\title{
On the potential of extrasolar planet transit surveys
}

\author{
M. Gillon ${ }^{1}$, F. Courbin ${ }^{2}$, P. Magain ${ }^{1}$, and B. Borguet ${ }^{1}$ \\ ${ }^{1}$ Institut d'Astrophysique et de Géophysique, Université de Liège, Allée du 6 Août, 17, Bat. B5C, Liège 1, Belgium \\ e-mail: gillon@astro.ulg.ac.be \\ 2 Laboratoire d'Astrophysique, École Polytechnique Fédérale de Lausanne (EPFL), Observatoire, 1290 Sauverny, Switzerland
}

Received 9 March 2005 / Accepted 20 July 2005

\begin{abstract}
We analyse the respective benefits and drawbacks of ground-based and space-based transit surveys for extrasolar planets. Based on simple but realistic assumptions about the fraction of lower main sequence stars harboring telluric and giant planets within the outer limit of the habitable zone, we predict the harvests of fictitious surveys with three existing wide field optical and near-IR cameras: the CFHT-Megacam, SUBARUSuprime and VISTA-IR. An additional promising instrument is considered, VISTA-Vis, currently under development. The results are compared with the harvests predicted under exactly the same assumptions, for the space missions COROT and KEPLER. We show that ground-based wide field surveys may discover more giant planets than space missions. However, space surveys seem to constitute the best strategy to search for telluric planets. In this respect, the KEPLER mission appears 50 times more efficient than any of the ground-based surveys considered here. KEPLER might even discover telluric planets in the habitable zone of their host star.
\end{abstract}

Key words. astrobiology - surveys - techniques: photometric - stars: planetary systems

\section{Introduction}

More than 160 extrasolar planets have been discovered ${ }^{1}$ since $^{2}$ the first detection of a "Hot Jupiter" around the main-sequence star 51 Peg (Mayor \& Queloz 1995). Most of these discoveries are the results of radial velocity (RV) searches. However, while RV searches are very efficient at detecting massive planets, they do not allow, on their own, to characterize completely the orbit of the planet. Due to the degeneracy between the mass of the planet and the inclination of its orbit with respect to the line of sight (see, e.g., Perryman 2000, for a review), $M \sin i$ is the only measurable quantity using the RV technique alone, where $M$ is the mass of the planet and $i$, the inclination of its orbit. At the end of 1999, a significant advance was accomplished by the observation of the first transit of an extrasolar planet discovered through the RV method (Henry et al. 2000; Charbonneau et al. 2000). With simultaneous spectroscopic and photometric observations of the star during the transit, both the mass and the radius of the companion object could be determined, confirming beautifully its planetary nature, and demonstrating the great interest of combining RV and transit observations.

Transit photometry is not only of interest as a follow up of RV searches, but also as a way to find new planets. It may prove even more sensitive to low mass planets than RVs. In fact, the method is considered as one of the most

1 See Extrasolar Planets Catalog, http: //www . obspm.fr/encycl/catalog.html promising ways of finding earth-class planets (Sackett 1999; Schneider 2000). The OGLE survey (Optical Gravitational Lensing Experiment, Udalski et al. 2002a,b), a ground-based experiment initially devoted to stellar microlensing in the galactic bulge, has been extended to the search for planetary transits. In 2003, Konacki et al. (2003a,b) announced the characterization of the first exoplanet discovered by transit photometry, orbiting the star OGLE-TR-56. The surprisingly short period of 1.2 day of the companion object was confirmed by RV follow-up, a value much below the lower end of the period distribution of planets detected by RV surveys (Udry et al. 2003). This important discovery demonstrated the effectiveness of transit photometry at detecting extrasolar planets, and was soon followed by four other discoveries by the same team (Bouchy et al. 2004; Pont et al. 2004; Konacki et al. 2005). Other surveys have started, such as TrES (Trans-Atlantic Exoplanet Survey) multisite transit survey, and its first discovery of an exoplanet, TrES-1 (Alonso et al. 2004). Very recently, transits of a saturnian size planet discovered through the RV method have been observed by the N2K Consortium (Sato et al. 2005) around the star HD 149026, leading to a total of eight stars known to experience planetary transits.

With the potential of the transit method now well established, space missions have been proposed in the hope to discover dozens of new planets. Among them are the COROT (Rouan et al. 2000) and KEPLER (Koch et al. 1998) missions, designed to perform high precision photometry of selected 
fields over several years. In parallel, wide field cameras are becoming available on large ground-based telescopes, making it possible to survey, from the ground, large numbers of stars simultaneously. The aim of the present paper is to weight the relative benefits and drawbacks of ground-based and space surveys, by carrying out a comparative study of their efficiency based on simple simulations. In particular, we investigate whether a large dedicated ground-based survey could be a competitive alternative to a high-cost space mission, or whether both approaches are complementary.

On the basis of realistic assumptions, we estimate the harvest in extrasolar planets for each type of survey. Our main goal is to compare the merits of each survey type, rather than predicting absolute discovery rates. Such absolute predictions are far too sensitive to the assumptions about the physical properties and about the formation process of planetary systems. We consider only simple distributions of planets around main sequence stars, in terms of radii and orbital distances. These are the only relevant quantities needed to carry out a comparative study, along with estimates of the density of stars in the simulated stellar fields.

The method used to estimate the harvests in planets is described in Sect. 2. The surveys considered and the results of our simulations are presented in Sect. 3, while the results and conclusions are summarized in Sect. 4.

\section{Description of the method}

\subsection{Strategy and field selection}

Planetary transits are rare and the eclipse seen in their host stars is extremely shallow: around $10^{-2}$ mag for a Jupiter-size planet and $10^{-4}$ mag for an Earth-size planet in orbit around of a solartype star. The question of the compromise that inevitably has to be made between field and depth is then a critical issue for any transit search. In addition, the time scales involved, i.e. the (expected) duration of the transits, is limiting the range of suitable exposure times, as soon as the goal is to properly sample the light curves during the transit phase. The maximum exposure for a given telescope is therefore mainly dictated by the size of the planets one wished to discover with respect to the size of its host star. Clearly, large telescopes are required in order to apply as short as possible exposure times and to sample the light curves at best. Wide field is also of major importance to observe many stars simultaneously and to compensate for the scarcity of transit events (e.g., Mallén-Ornelas et al. 2003). Being aware of this general line to design surveys, we describe in the following the simulations used in order to estimate the harvests of fictitious and real planetary transit surveys.

\subsection{Suitable host stars}

\subsubsection{Density and spectral types}

For a given planetary radius, the transit depth is inversely proportional to the square of the stellar radius. Thus, planetary transits will be more easily detected in cool dwarf stars. In this paper, we only consider the main-sequence stars with spectral

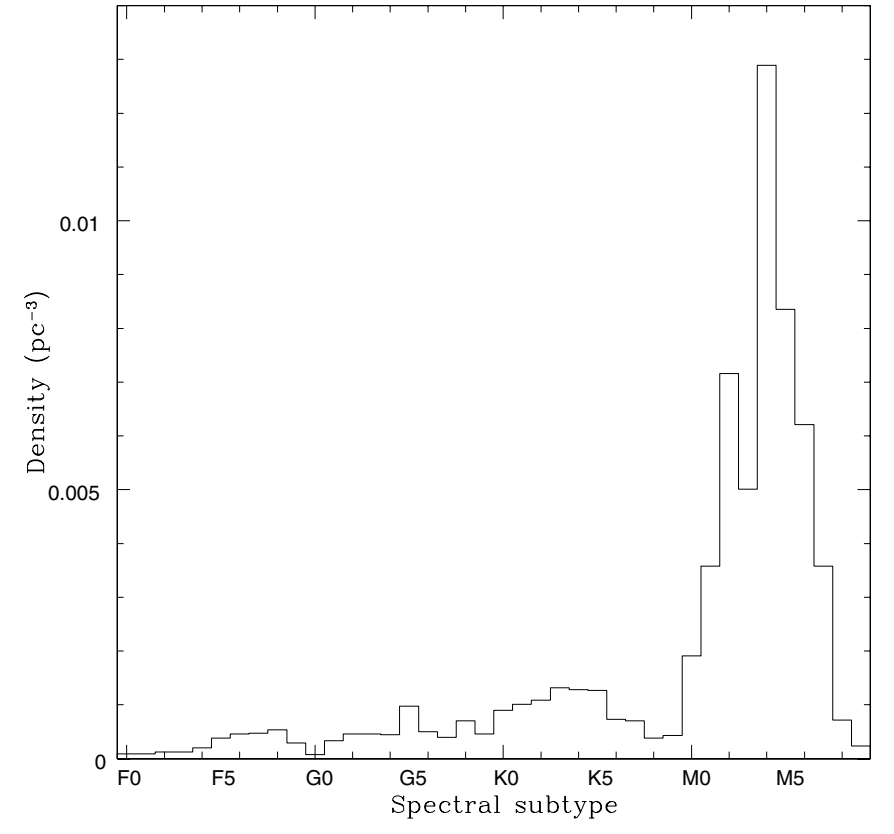

Fig. 1. Density of Lower Main Sequence Stars (LMSS) spectral subtypes in the solar neighborhood, estimated from the Gliese (1991) and Zakhozhaj (1979) nearby stars catalogues.

subtypes from $F 0$ to $M 9$ as suitable candidates. We refer to them as Lower Main-Sequence Stars (LMSS).

The galactic plane is the obvious place to look at, in order to observe simultaneously many LMSS. It is, indeed, the strategy adopted in the pioneering work by Mallén-Ornelas et al. (2003). Since the highest possible Signal-to-Noise Ratio (SNR) per exposure is required, fields with minimal reddening are prefered. A best choice consists of fields close to, but not right in, the galactic plane, with galactic latitudes between $2^{\circ}$ and $6^{\circ}$. We have chosen to carry out all our computations for fields with a mean latitude of $4^{\circ}$, where a representative extinction coefficient is $A_{\mathrm{V}}=0.7 \mathrm{mag} / \mathrm{kpc}$ (Schlegel et al. 1998).

The projected densities of $L M S S$ at low galactic latitude are estimated from the Gliese \& Jarheiss (1991) and the Zakhozhaj (1979) catalogues of stars in the solar neighborhood (see Fig. 1). We assume that these stellar densities also apply to the rest of the galactic plane. This is probably a fair approximation as long as the galactic bulge is avoided. The fields we are modeling here mainly contain stars belonging to the spiral arms of our Galaxy.

Under these assumptions, the number of stars in the field of view is computed for every half-magnitude bin and for all spectral subtypes from $F 0$ to $M 9$. The volumes sampled by the Gliese et al. (1991) and Zakhozhaj et al. (1979) catalogues are scaled to the volumes of each of the surveys considered. Thus, the total number of stars observed per half-magnitude bin depends on the size of the field of view and the filter used. Figure 2 shows the distribution of $L M S S$, computed for all subspectral types and for 4 limiting magnitudes in the $I$-band. Going to the infrared allows to observe a larger fraction of verylow-mass stars, as is shown in Fig. 3. 

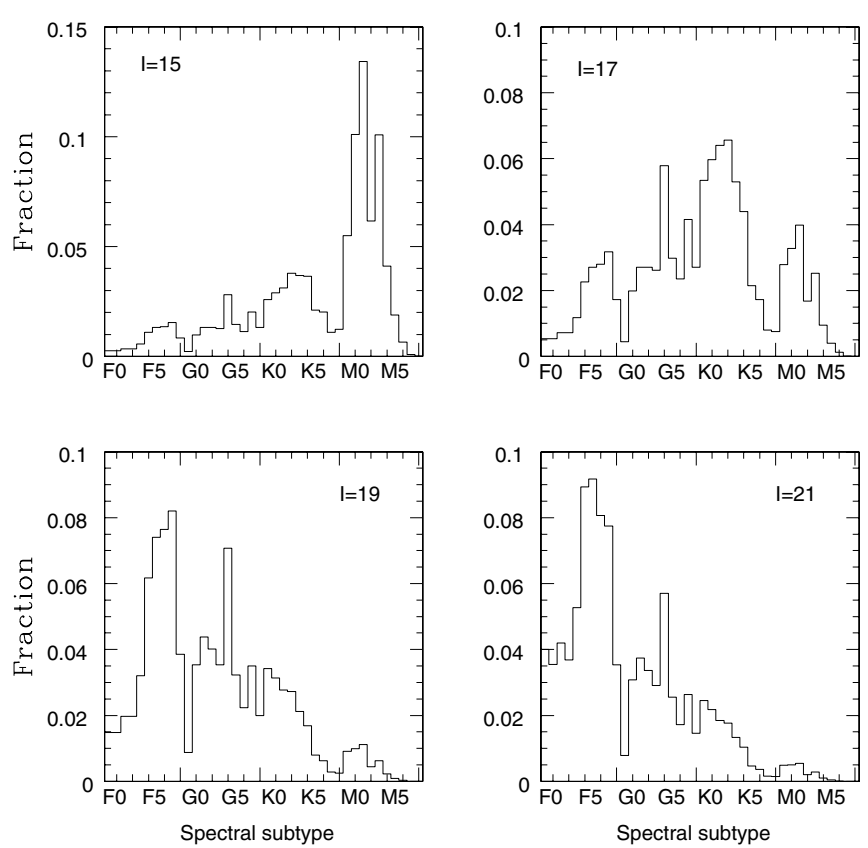

Fig. 2. Spectral subtypes distribution of LMSS for 4 limiting magnitudes $\left(15,17,19\right.$ and 21). An extinction coefficient $A_{\mathrm{V}}=0.7 \mathrm{mag} / \mathrm{kpc}$ and a maximum distance $D_{L}=4300 \mathrm{pc}$ are used (see text).

\subsubsection{Blends and binarity}

All our calculations are exclusively made for $L M S S$ and do not include the effect of blends, for two reasons:

1. Introducing all spectral types will increase the number of potentially interesting transits. It will also increase the crowding of the fields. Since stars other than LMSS have larger diameters, the net result of the competition between the two effects is likely a reduction of the true observable number of transits. Our results are therefore upper limits on the number of transits seen by each survey. However, since our goal is a comparative study between surveys, rather than an attempt to predict absolute transit counts, leaving aside the spectral types other than $F 0$ to $M 9$ does not affect our conclusions.

2. More blends also mean an increased number of false detections. For Jovian-mass planets, a large part of these false detections can be rejected through a fine-tuned transit light-curve analysis or with follow-up RV observations (Bouchy et al. 2005). In some cases, deciding whether an eclipse is due to a genuine planet or not can be very tricky (see e.g. Mandushev et al. 2005). The difficulty increases drastically in the case of a telluric planet. It does not only depend on the planet type, but also on the method of analysis used to build the stellar light curves and on the transit detection method and selection criteria: methods based on aperture photometry will be heavily affected by seeing and blends, while differential imaging, PSF fitting, or image deconvolution behave very differently with respect to blends and to PSF mismatch. Finally, prior knowledge on the exact field geometry can be introduced as well in the analysis, e.g., by targetting fields with archived HST images used as reference maps to carry out the photometry. The degree of
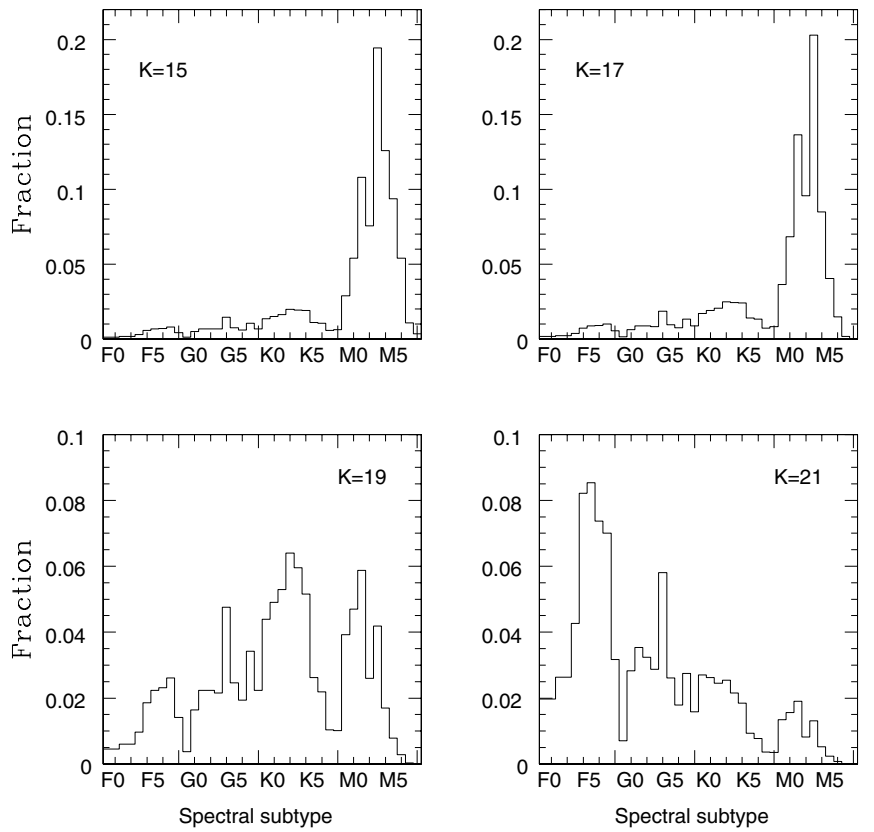

Fig. 3. Same figure as Fig. 2, but in the $K$-band. The proportion of very-low-mass stars, for a given limiting magnitude, is larger than in the $I$-band.

additional complication due to blends is such that it falls well out of the scope of the present paper. Again, only relative estimates between surveys are aimed at.

Very little is known about the formation of planets in binary systems. Furthermore, detecting transits in close binary systems is possible but can be difficult, because of the decrease of the transit depth. We have chosen to adopt an arbitrary fraction of 50\% of binary LMSS and to do not allow a transit detection around them. The exact value of this fraction has no influence on the result of our comparative study. Our whole simulations are designed to predict the maximum number of planets that can potentially be discovered with each type of survey, with a slight and arbitrary correction for binarity.

\subsubsection{Distance}

Among our assumptions is the maximum distance above which the density of LMSS may not be valid. As we consider fields with a low but non-zero galactic latitude, this maximum distance can be computed from the thickness of the galactic disk. Considering a thin disk with a thickness of $600 \mathrm{pc}$, positioning the Sun at mid height, and using an average galactic latitude of $4^{\circ}$ for all the surveys considered in this work, the limiting distance $D_{\mathrm{L}}$ is simply:

$D_{\mathrm{L}}=\left(\frac{600}{2}\right) \times\left(\frac{1}{\sin 4^{\circ}}\right) \approx 4300 \mathrm{pc}$.

In the following we will consider that the density of $L M S S$ drops to zero beyond the distance $D_{\mathrm{L}}$. The limiting magnitudes imposed to us by the telescopes and cameras chosen in this work for ground-based surveys are well above the limiting magnitude due to pure distance effects. The magnitude range is 

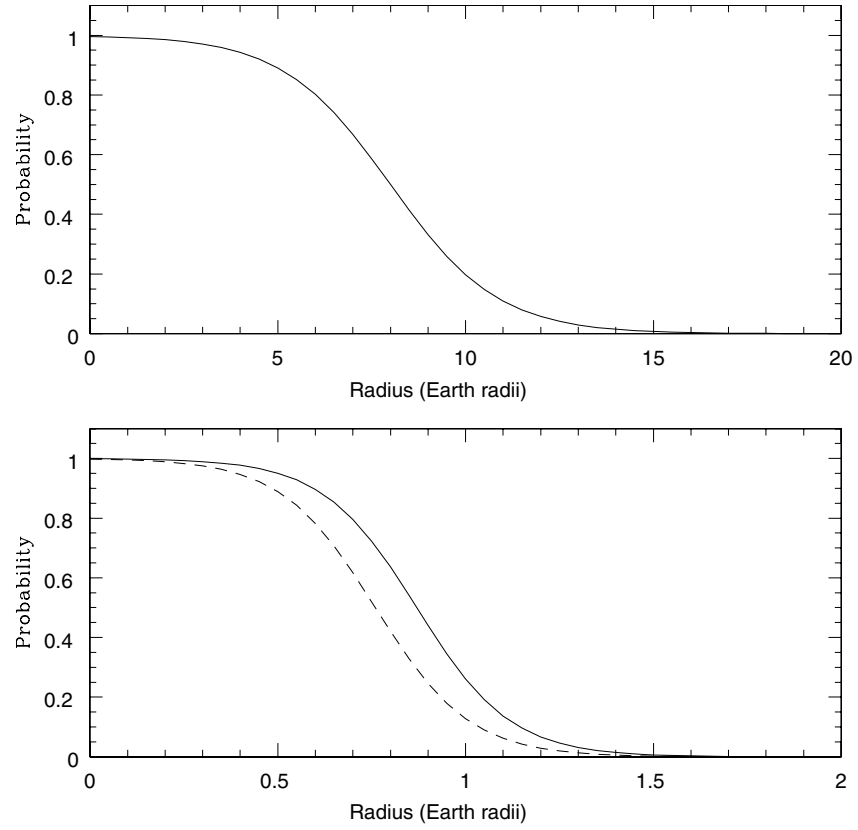

Fig. 4. Top: radii distribution of giant planets interpolated from the four giant planets in our Solar System. The $y$-axis gives the probability that a planet has a radius larger than the value on the $x$-axis, in earth radii. Bottom: radii distribution of $T H Z$ (solid line) and $T I Z$ (dotted line), interpolated from the simulations of the formation of telluric planets by Chambers (2001).

therefore limited by distance rather than by instrumental depth. For space-based surveys, the instrumental depth is the limiting factor.

\subsection{Estimated number of planets in the field}

Once the number of target stars has been estimated, one also needs to make assumptions about the average number of planets hosted by LMSS, and on the distribution of their radii and periods. We consider six types of planets:

- Very Hot Jupiters $(V H J)$ : we define these objects as giant planets with a maximum period of 3 days and an arbitrary minimum period of 1 day. On the basis of the results of Gaudi et al. (2005), we assume that $0.2 \%$ of LMSS may host a $V H J$. We use a rough radii distribution represented by a sigmoid function, interpolated from the radii distribution observed in our own solar system (see Fig. 4). We define the $V H J$-Zone as the range of distances to the host star corresponding to orbital periods between 1 and 3 days. We assume that the periods of the $V H J$ are uniformly distributed in the $V H J$-Zone.

- Hot Jupiters $(H J)$ : are defined as giant planets with a period in between 3 and 9 days. Following Gaudi et al. (2005), we adopt a value of $1 \%$ for the fraction of $L M S S$ orbited by such objects. We use the same radii distribution as for the $V H J$. We define the $H J$-Zone as the region around a star where the period of a planet is between 3 and 9 days. As noticed by Gaudi et al. (2005), about half of the $H J$ have a period lower than 3.5 days. We thus consider that half of the $H J$ have periods uniformly distributed in the range
3-3.5 days, and that the other half have periods uniformly distributed in the range 3.5-9 days. $H J$ and $V H J$ forms the group of the Close - in Extrasolar Giant Planets (CEGP). We shall denote them $(V) H J$ when considered as a single group.

- Giant planets in the Habitable Zone (GHZ): giant planets in the zone identified by Kasting et al. (1993) as suitable for life. We use the limits established by Kasting et al. (1993) for the Zero Age Main Sequence $H Z$ (ZAMS HZ) and their "intermediate" habitability criteria. Based on the measured fraction of extrasolar planets found in the $H Z$, we assume that $3 \%$ of the LMSS harbor such a planet. We use the same radii distribution for these objects as for the $H J$ and $V H J$. We consider that the periods of the $G H Z$ are uniformly distributed across the $H Z$.

- Giant planets in the Intermediate Zone (GIZ): the Intermediate Zone is located between the $H Z$ and the $H J$-Zone. We assume that the fraction of LMSS with a GIZ is the same as in the $H Z$, i.e., $3 \%$. The same radii distribution is used for $G I Z$ as for the other types of giants planets considered. We consider that the periods of the $G I Z$ are distributed uniformly in the Intermediate Zone.

- Telluric planets in the Habitable Zone ( $T H Z$ ): nothing is known about telluric planets outside our own Solar System. We base our estimates on the terrestrial planets formation simulations of Chambers (2001). Using these results and the assumption that all LMSS are surrounded by a protoplanetary disk at the beginning of their life (which will lead to the formation of telluric planets) we obtain 1.78 as the average number of terrestrial planets in the $H Z$ per $L M S S$. This number does not take into account the fact that, for a poorly constrained fraction of the stars, one or more giant planet may migrate inwards over the outer limit of the $H Z$, hence affecting the orbits of the telluric planets and maybe accreting them or expulsing them on a hyperbolic orbit. We take this fraction as the sum of the fractions of $V H J, H J$, $G H Z$ and $G I Z$, leading to $7 \%$ of the LMSS hosting a giant planet inside the outer limit of the $H Z$. Assuming that no terrestrial planet can survive under these conditions, we obtain $1.78 \times(1-0.07) \approx 1.66$ as the average number of terrestrial planets in the $H Z$ per $L M S S$. We also use the results of Chambers (2001), interpolated with a sigmoid function (see Fig. 4) to estimate the radii distribution of telluric planets in the $H Z$. We consider that the periods of the $T H Z$ are distributed uniformly in the $H Z$.

- Telluric planets in the Intermediate Zone (TIZ): using the results of Chambers (2001) and correcting for the fraction of LMSS having a giant planet inside the outer limit of the $I Z(4 \%)$, we estimate the average number of terrestrial planets in the $I Z$ to be 1.51 per $L M S S$. The results of Chambers are again used to estimate the radii distribution of telluric planets in the $I Z$ (Fig. 4). We consider that the periods of the $T I Z$ are distributed uniformly in the $I Z$.

The planets outside the $H Z$ are not considered here, as their long period and their low geometric probability of producing a detectable transit make them very poor candidates. We also do not consider telluric planets with a period shorter than 9 days, 
Table 1. Instruments considered in our fictitious ground-based surveys.

\begin{tabular}{lccr}
\hline \hline Instr. & $\begin{array}{c}\text { Telescope } \\
\text { diameter }\end{array}$ & $\begin{array}{c}\text { Field } \\
\text { (sq. degrees) }\end{array}$ & Location \\
\hline Megacam & $3.6 \mathrm{~m}$ & 0.90 & Hawaii (CFHT) \\
Suprime & $8.3 \mathrm{~m}$ & 0.26 & Hawaii (Subaru) \\
Vista-IR & $4.0 \mathrm{~m}$ & 1.00 & Chile (VLT) \\
Vista-Vis & $4.0 \mathrm{~m}$ & 2.25 & Chile (VLT) \\
\hline
\end{tabular}

because they are not predicted by the simulations of Chambers and because there is no such planet in our own solar system.

Our assumptions about giant planets are empirical, and based on previous surveys that are certainly biased towards a given type of planet and orbit and still suffer from low number statistics. Furthermore, we assume that the average expected number of planets for the four zones we defined earlier are the same for all spectral subtypes from $F 0$ to $M 9$. This assumption does not have a strong influence on the results given our already rough knowledge of planetary formation, although a metallicity dependence on exoplanets population has been emphasized (see e.g. Santos et al. 2004) and low-mass stars tend to be older on average and have a lower metallicity. The predicted harvests should nevertheless be of the right order of magnitude and are certainly adequate for comparison purposes.

The assumptions made on telluric planets are based on a purely theoretical work. We do not take into account the possible existence of giant planet cores formed in the outer region of the disk and that later migrated inwards. These objects, without a massive primary atmosphere, could consist of large planets of pure rock and ice. We prefer to remain conservative and to consider exclusively planets formed in the more standard scenario thought to be responsible for the formation of planetary systems such our own.

The known weaknesses of the assumptions used in our simulations are not a critical issue for our purpose, which is to compare the relative strengths and weaknesses of different transit surveys rather than to predict actual discovery rates for a given survey. Our results may be scaled up and down, but we believe that they remain correct as long as the aim is to carry out comparative studies.

\subsection{Parameters of the instruments chosen for the fictitious ground-based surveys}

Transit searches must combine large field of view, depth and good temporal sampling. Although the range of possible telescope/camera combinations is broad, we have selected four instruments that can be considered as representative of the present or soon available astronomical facilities. Three of these instruments are already in use: CFHT-Megacam, Subaru-Suprime, and Vista-IR. The latter one is only proposed: Vista-Vis $^{2}$ (see Table 1).

For each instrument, we have estimated the $S N R$ and the saturation magnitude for a range of exposure times, for a fixed

\footnotetext{
2 See VISTA web site, http://www.vista.ac.uk
}

airmass (1.6) and typical seeing ( 1 arcsec). These parameters are computed for different filters, using the Exposure Time Calculators (ETC) available for Subaru and the CFHT. The bright cut applied to the magnitude distribution of host stars corresponds to saturation time. The faint cut corresponds to the magnitude of stars that have a $S N R<10$, where a shallow transit would not be detected.

For Vista-Vis, we used the information given in the online ETC of the EMMI camera. This ESO instrument is mounted on the $3.5 \mathrm{~m} \mathrm{NTT}$ at La Silla (Chile) and has a throughput similar to Vista, which will also be a 4 m-class telescope. For Vista-IR, we use the information given on the online ETC of the SOFI IR camera of the ESO $3.5 \mathrm{~m}$ NTT.

All our estimated $S N R$ are corrected for the extra source of noise introduced by stellar variability, following the formula:

$S N R_{2}=\frac{S N R_{1}}{\sqrt{1+\left(\sigma_{*} S N R_{1}\right)^{2}}}$

where $S N R_{1}$ and $S N R_{2}$ are the $S N R$ before and after taking $\sigma_{*}$ into account, the standard deviation of the stellar variability. We adopt $\sigma_{*}=100 \mathrm{ppm}$ as estimated from the solar variability within a frequency interval that matches our adopted range of transit durations (Bordé 2003). We consider this same value for every $L M S S$ spectral subtypes.

\subsection{Computation of the harvests and detection criteria}

Under all the above assumptions, the expected numbers of transit detections are estimated as follows.

For every spectral subtype F0 to M9, we compute 100 circular orbits for each of the four zones considered, distributed as mentioned in Sect. 2.3. The geometric transit probability for an orbit is given by

$P_{\mathrm{tr}}=\frac{R_{*}}{a}$

where $R_{*}$ is the radius of the star and $a$ the semi-major axis of the planet orbit. For every orbit and for a range of inclinations and planetary radii, we compute the total crossing-time of the transit and the duration of the flat part of the light curve, following the calculation by Mallén-Ornelas et al. (2001). The results are then averaged on the inclinations and radii.

A window function is computed for each survey, based on the total number of nights in the campaign and the visibility of the field each night, in the case of ground-based surveys. To take weather effects into account, we assume that for 1 night out of 10, no observations are taken at all. In addition, 10 chunks of $1 \mathrm{~h}$ are randomly removed from the 9 remaining nights to account for technical problem, clouds, or unexpected overheads.

We then compute a probability $P_{\text {vis } N}$ that a transit is observed $N$ times for a specific orbit and a specific LMSS. We compute this probability for $N$ varying from 1 to $X$, where $X$ is the maximum number of transits we could observe for the same star during the whole observing season. As the shortest period considered is 1 day, $X$ is simply the duration of the survey in days. 
For a specific spectral type and a specific distance, the probability $P_{\text {obs } N}$ that a transit occurs $N$ times during the survey and is observable is $P_{\mathrm{obs} N}=P_{\mathrm{tr}} \times P_{\mathrm{vis} N}$.

We then consider that the observed dimming of a light curve can be attributed to a genuine planet only if at least three eclipses are detected. We define $k$ as the number of transits observed during the survey and impose that the $S N R$ of the light curve, integrated on the duration of the flat part of the $k$ transits is at least $\beta$ time greater than the inverse of transit depth, i.e.

$S N R \geq \frac{\beta}{\sqrt{k}}\left(\frac{R_{*}}{R_{p}}\right)^{2}$,

where $R_{p}$ is the planetary radius and $R_{*}$ the radius of the host star.

In other words, the significance of planetary transits in a stellar light curve is $\beta \times \sigma$.

We have chosen to adopt $\beta=9$. This value is high enough to reject the majority of the statistical artifacts. Is is also the one adopted by the OGLE-III team (Udalski, private communication). The value $\beta=7$ is also tested, as it is used in other transit studies such as in Bordé et al. (2003) for the COROT mission.

For a specific planet transiting $k$ times during the survey, and for a star of a given magnitude and a given radius $R_{*}$, Eq. (4) allows a computation of the minimum planetary radius needed to get a $S N R$ high enough to allow a statistically significant detection. The relevant radii distribution then leads to a determination of the probability that the planet has a radius at least equal to the minimum radius of detection, i.e. the fraction of planets which would produce a dimming strong enough to be detected.

For every half magnitude bin and for every zone considered, the results are averaged over 100 orbits and multiplied by the number of stars of each spectral type present in each halfmagnitude bin. We then multiply by the fraction of stars expected to host a planet (giant or telluric) in the zone considered, leading to the expected number of planet detections. As a final step, we sum the numbers of planets for each half-magnitude bin and spectral subtype, and obtain the total number of detections for each planet type.

\section{Results}

\subsection{Analysis of existing surveys: OGLE-III and EXPLORE-I}

We have tested our simulations on two existing groundbased surveys, EXPLORE-I (Mallén-Ornelas et al. 2003) and OGLE-III (Udalski et al. 2002a,b, 2004). The goal was to compare our predictions to the actual results of these surveys and to check the validity of our assumptions. Our main goal remains the comparison of existing ground-based surveys to future fictitious ground-based surveys and to space missions.

\subsection{EXPLORE-I}

The Extrasolar Planet Occultation Research search lasted 11 nights, at the CTIO $4 \mathrm{~m}$ telescope, in the $I$-band with the
Table 2. Results predicted for EXPLORE-I. In the first line, we adopt $P_{\text {vis2 }}=0.1$ for $(V) H J$ and $P_{\text {vis2 }}=0$ for the other types of planets. The second and third lines involve a $P_{\mathrm{visN}}$ computed assuming good weather conditions (see text). The values in brackets are for $\beta=7$, and the others correspond to $\beta=9$.

\begin{tabular}{ccc}
\hline \hline Weather conditions & $V H J$ & $H J$ \\
\hline Real conditions $(k=2)$ & $0.4(0.5)$ & $1.2(1.5)$ \\
Good weather $(k \geq 2)$ & $3.4(4.2)$ & $2.8(3.4)$ \\
Good weather $(k \geq 3)$ & $2.2(2.7)$ & $0.7(0.9)$ \\
\hline
\end{tabular}

mosaic II camera. It concentrated on one single $0.36 \mathrm{deg}^{2}$ field near the galactic plane $\left(l=-27.8^{\circ}, b=-2.7^{\circ}\right)$ containing $\sim 100000$ stars down to $I=18.2$ and $\sim 350000$ stars down to $I=21.0$. Poor weather conditions led to a degraded window function, affecting the probability $P_{\text {vis } 2}$ to detect two transits of the same planet. $P_{\text {vis2 }}$ was estimated as 0.1 for $(V) H J$ by the EXPLORE team (Mallén-Ornelas, private communication).

The typical exposure time was $60 \mathrm{~s}$, and the detector read time plus overhead amounted to $101 \mathrm{~s}$. The analysis of the data is not completed, and no final detection criterion has been decided up to now (Mallén-Ornelas, private communication). No exoplanet transit has been discovered so far.

Following the simulations presented in this paper, with $P_{\text {vis2 }}=0.1$ for $(V) H J$ (and $P_{\text {vis } N>2}$ set to 0 , i.e. assuming that the probability to detect three transits of the same planet is negligible), we compute the expected harvest for EXPLORE-I, given its true weather conditions. We also compute the harvest for good weather conditions, i.e. without fixing $P_{\mathrm{vis} N}$ but estimating it according to the method described in Sect. 2.5. Our results for the two detection criteria $(\beta=9$ and $\beta=7)$ are presented in Table 2.

We present in Fig. 5 the magnitude distribution of the host stars $(V) H J$ expected according to our simulation under good weather conditions and $\beta=9$. Figure 5 also shows the distribution of the distances, of the spectral subtypes and of the number of transits observed for the expected discoveries.

Mallén-Ornelas et al. (2001) expected to find 1 transiting $(V) H J$. Although this is very low number statistics, this value is in good agreement with our result for $P_{\text {vis2 }}=0.1$. The absence of any detection is also compatible with our estimates. We point out that good weather conditions could have led to the discovery of a few $(V) H J$, showing that searches using $4 \mathrm{~m}$-class telescopes and a wide-field camera are promising strategies. EXPLORE-I lasted only 11 nights. A simple scaling of the results for a 3-months survey shows that a harvest of several tens of $(V) H J$ is not unrealistic.

\subsection{OGLE-III}

The Optical Gravitational Lensing Experiment entered its third phase, OGLE-III, in June 2001. It took place at the Las Campanas Observatory, Chile, using the $1.3 \mathrm{~m}$ Warsaw telescope and the 8k MOSAIC camera, with a total field of view 

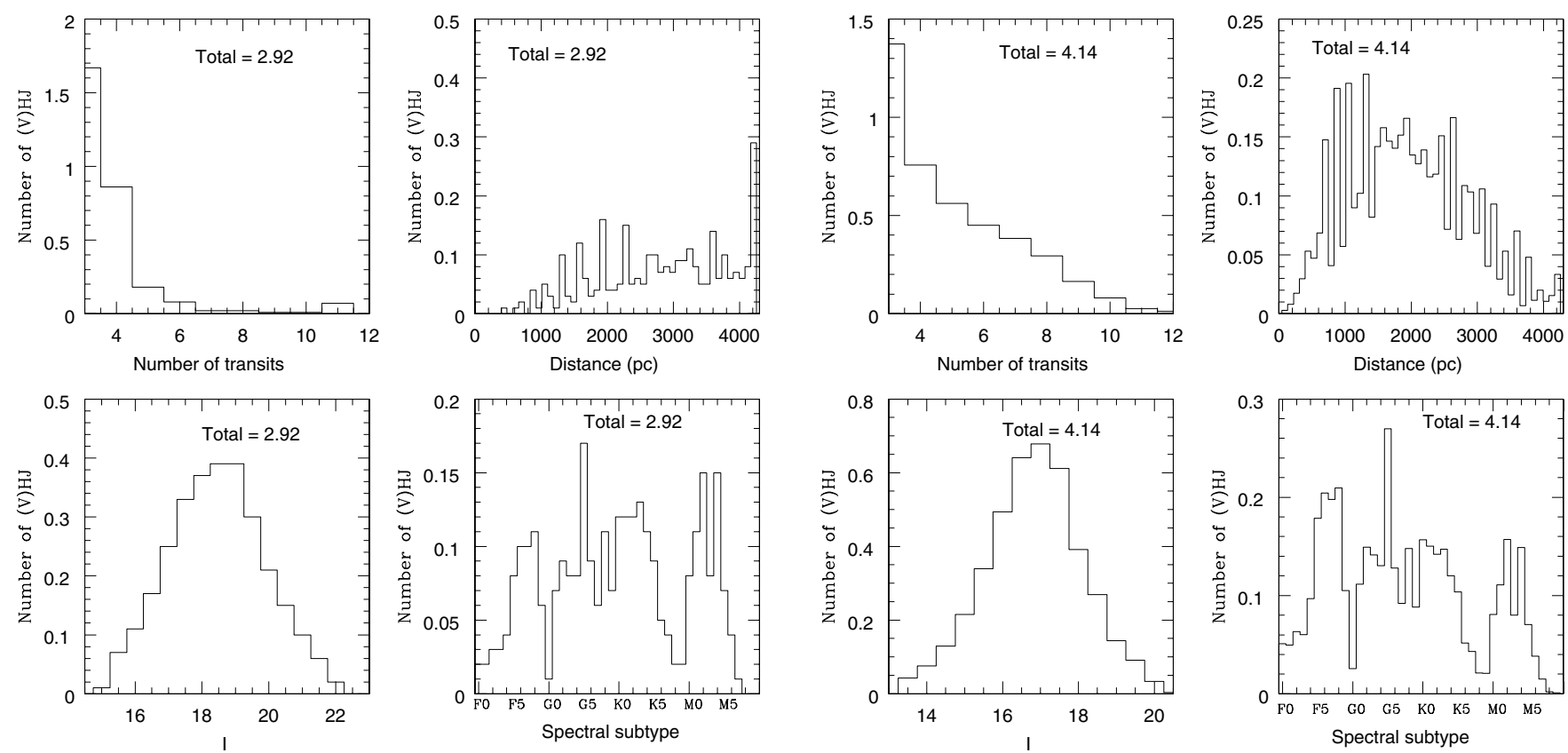

Fig. 5. Distribution of the expected number of $(V) H J$ for EXPLORE-I (with good weather conditions) as a function of the number of transits observed (upper left), of the distance (upper right), the magnitude (bottom left) and the spectral subtype (bottom right) of the host star. The irregular shape of the histogram as a function of distance is an artificial effect caused by the rounding off of the stellar magnitudes to the nearest integer or half-integer.

of $0.34 \mathrm{deg}^{2}$. All observations were made through the $I$ filter. Four surveys have been carried out so far:

- OGLE-III-1 (June 12 to July 28, 2001). More than 800 images of three fields in the direction of the galactic bulge were collected within 32 nights. The exposure time was $120 \mathrm{~s}$, and each field was observed every $12 \mathrm{~min}$.

- OGLE-III-2 (February 17 to May 22, 2002). More than 1100 images of three fields located in the Carina region of the galactic disk were collected in 76 nights. The exposure time was $180 \mathrm{~s}$, and the temporal resolution was about $15 \mathrm{~min}$.

- OGLE-III-3 (February 12 to March 26, 2003). The photometric data were collected during 39 nights spanning the 43 days of the survey. Three fields of the galactic disk were observed with a time resolution of about $15 \mathrm{~min}$. The exposure time was $180 \mathrm{~s}$.

- OGLE-III-4. Starting on March 25, 2003, this survey collected its main photometric material until middle May 2003, but observations were also collected until July 25, 2003 with sparse temporal sampling. Three fields of the galactic disk were observed with the same exposure time and resolution as the two previous surveys.

Using the observation windows and instrumental parameters of these 4 surveys (A. Udalski, private communication), we have computed the expected harvests for the two detection criteria (see Table 3). OGLE-III-1 and OGLE-III-2 have yield 5 genuine extrasolar planets so far. OGLE-III-3 and OGLE-III-4 have discovered 40 transiting companions, but follow-up

Fig. 6. Distribution of the $(V) H J$ discoveries for OGLE-III-2 as a function of the number of observed transits (upper left), distance (upper right), magnitude (bottom left) and of the host star spectral subtype (bottom right).

Table 3. Results predicted by our simulations for OGLE-III surveys, compared to the actual harvests. The values are given for $\beta=9$ and the values in parenthesis correspond to $\beta=7$. No actual harvest is available yet for OGLE-III-3 and -4 .

\begin{tabular}{cccc}
\hline \hline Survey & Planet & Predicted & Actual harvest \\
\hline OGLE-III-1 & VHJ & $1.6(2.2)$ & 1 \\
& $H J$ & $0.7(0.9)$ & 1 \\
OGLE-III-2 & VHJ & $2.7(3.6)$ & 2 \\
& $H J$ & $1.5(2.0)$ & 1 \\
OGLE-III-3 & $V H J$ & $2.1(2.8)$ & - \\
& $H J$ & $0.8(1.1)$ & - \\
OGLE-III-4 & $V H J$ & $2.6(3.6)$ & - \\
& $H J$ & $1.8(2.5)$ & - \\
\hline
\end{tabular}

spectroscopy has not given the final harvest yet. We note that, although predicting absolute planet counts is not our main goal, our OGLE-III expected harvests are in very good agreement with the actual ones. Our work hypotheses thus allow to compute realistic harvests in the case of $(V) H J$.

Figure 6 shows the distribution of the $(V) H J$ discoveries predicted by our simulation for OGLE-III-2 as a function of the characteristics of the host star and of the number of observed transits. Most detections occur for stars located between 1500 and $2500 \mathrm{pc}$ from the Sun, well matching the range of distances for the real planets OGLE-TR-111, OGLE-TR-113 and OGLE-TR-132, i.e., about 1500 pc (Schneider, Extrasolar Planet Catalog). We also note that our predicted ratio of $V H J$ to $H J$ is in good agreement with the observed one (see Table 3). Future planet harvests with OGLE-III-3 and OGLE-III-4 shall allow to better constraint the absolute counts of $V H J$ around $L M S S$. 


\subsection{Analysis of fictitious surveys}

\subsubsection{Setting the parameters of the surveys}

All the surveys we consider in the following use red filters, i.e., redder than the $V$-band. This choice is motivated by the fact that limb darkening and atmospheric and galactic absorptions are minimized in the red. It also maximizes the number of available $L M S S$, hence increasing the probability of detecting extrasolar planets. Extending the above argument from the visible to the near-infrared, we have included surveys using the $J$, $H$ and $K$ filters, on VISTA-IR. However, we have not included the longer wavelength filters such as $L$ or $M$, because the quantum efficiency of the detectors is much lower with present-day instrumentation.

The choice of the exposure time is a critical issue for surveys carried out in the visible. Long exposure times lead to high $S N R$, but also to a far too large number of saturated stars in galactic fields. Increasing the exposure times also increases crowding, and consequently decreases the SNR for any contaminated star. As mentioned in Sect. 2, effects of crowding are not taken into account here, and will be the subject of a forthcoming paper. If the goal of the survey is to discover planets as small as possible, a compromise has to be found between the duration of the exposure time plus readout time of the CCD, the desired $S N R$ in each individual exposure and the number of sampling points during an eclipse. We have estimated the exposure time leading to the largest number of unsaturated $L M S S$ with sufficient $S N R$ and chosen to test three different exposure times close to this "optimal" value for each optical instrument. The actual readout time of each instrument is also taken into account in our calculations.

The situation is simpler for near-IR instruments, since the readout time is negligible. The harvest is thus largely insensitive to the adopted exposure time and we have adopted the exposure time leading to the largest number of unsaturated $L M S S$.

Finally, we have tested surveys of four different durations: $30,60,120$, and 180 nights.

We have checked that there exist fields in the galactic disk that can be observed continuously during six months, either from Paranal or Mauna Kea. However, their visibility is reduced to about $4 \mathrm{~h}$ during the first and the last month of a six months period. We have used the seasonal observability of typical fields of the galactic disk to build our window function and we have also investigated the interest of extended surveys, carried out over several years (between 1 and 4 years), similarly to space missions such as COROT and KEPLER, but with noncontinuous visibility.

The predicted harvests for the different surveys are summarized in Table 4. The columns in all tables are labeled as exposed in Sect. 2.

\subsubsection{Results}

In the following, we comment on the results presented in Table 4.

- Optical filters: optical surveys are about twice as efficient in the $I$-band as in the $R$-band. This is mainly due to the increased brightness of most LMSS in the $I$ filter, which more than compensates for the lower throughput of the instruments in the $I$-band. For this reason, we consider only I-band searches for surveys longer than 1 month.

- Near-IR filters: infrared surveys are more efficient in the $J$ and $H$-bands than in the $K$-band. In the infrared, the gain in the number of LMSS observed in $K$ does not compensate for the lower sensitivity of the detectors at this wavelength. The $J$ filter appears to be the best choice.

- Exposure times: in the optical, the longer exposure times do not always yield to larger harvests. Although this increases the SNR across the duration of the transit, it also leads to more saturated stars, so that the improvement in the harvest is negligible.

- (V)HJ vs. IZ planets: even for the shortest survey duration (30 days) and for the least favorable detection criterion $(\beta=9)$, the harvests in $(V) H J$ are always large, with tens of discoveries. Increasing the duration of the survey does not increase very much the number of $V H J$ detected, but increases drastically the number of giant planets found in the $H Z$ and in the $I Z$, the largest gain being for surveys longer than one year. This is simply due to the better matching of the time base-line with the longer revolution periods of planets in the $H Z$ and the $I Z$.

- Field vs. depth in the optical: among the existing optical instruments, the harvests obtained with SUBARU are smaller than those obtained at the CFHT, despite the larger telescope size: the improved depth of SUBARU does not compensate for the smaller field of view.

- Telluric planets: under the assumptions used here, our fictitious ground-based surveys seem to have a low potential for discovering TIZ. VISTA-Vis has the highest potential for this purpose, followed by VISTA-IR. Habitable planets seem out of the reach of ground-based surveys. The telluric planets which could be discovered by VISTA-Vis or VISTA-IR would probably orbit around M-dwarfs relatively close to the Sun (see Figs. 7 and 8).

\subsection{Expected harvest of the COROT mission}

COROT (COnvection, ROtation and planetary Transit) will be the first satellite launched with the aim of detecting exoplanets using the transit method (Rouan et al. 2000). Another goal of this mission is to carry out several projects in asteroseismology (Baglin et al. 1998). It uses a $27 \mathrm{~cm}$ telescope in combination with two $2048 \times 2048$ CCD cameras, and a final field of view of $3.5 \mathrm{deg}^{2}$. During the 2.5 years of the mission, 5 fields in the galactic plane will be observed continuously, each one during 150 days. The exoplanet detection capability of this mission has already been analysed (Bordé et al. 2003) but, since our goal is a comparison between surveys, we analyse the planet harvest expected from COROT using the same assumptions as for the other surveys considered.

COROT will observe fields at low galactic latitudes, i.e., in directions with a high density of LMSS. Indeed, each field will contain up to 12000 dwarf stars with visual magnitudes 
Table 4. Results obtained for fictitious surveys. $\mathrm{ET}=$ exposure + readout time. The values are for $\beta=9$. The values given between parenthesis correspond to $\beta=7$.

\begin{tabular}{|c|c|c|c|c|c|c|c|c|c|}
\hline Instr. & Band & Years/Nights & ET $(s)$ & $V H J$ & $H J$ & GIZ & $G H Z$ & $T I Z$ & $T H Z$ \\
\hline \multirow[t]{6}{*}{ CFHT } & $i^{\prime}$ & $1 / 30$ & 72 & $13.0(15.2)$ & $14.6(17.6)$ & $0.1(0.1)$ & - & - & - \\
\hline & & & 102 & $15.3(17.8)$ & $17.5(20.7)$ & $0.1(0.2)$ & - & - & - \\
\hline & & & 162 & 16.9(19.6) & $19.4(23.0)$ & $0.2(0.2)$ & - & - & - \\
\hline & $r^{\prime}$ & $1 / 30$ & 72 & $8.6(10.0)$ & $9.6(11.3)$ & $0.0(0.1)$ & - & - & - \\
\hline & & & 102 & $9.8(11.3)$ & $10.9(12.8)$ & $0.1(0.1)$ & - & - & - \\
\hline & & & 162 & $10.6(12.2)$ & $11.9(13.9)$ & $0.1(0.1)$ & - & - & - \\
\hline \multirow[t]{6}{*}{ SUBARU } & $I$ & $1 / 30$ & 70 & $4.7(5.4)$ & $5.5(6.4)$ & - & - & - & - \\
\hline & & & 90 & $5.8(6.5)$ & $6.9(7.8)$ & $0.1(0.1)$ & - & - & - \\
\hline & & & 120 & $6.1(6.9)$ & $7.3(8.4)$ & $0.1(0.1)$ & - & - & - \\
\hline & $R$ & $1 / 30$ & 70 & $3.5(4.0)$ & $4.1(4.7)$ & - & - & - & - \\
\hline & & & 90 & $4.1(4.6)$ & $4.8(5.4)$ & - & - & - & - \\
\hline & & & 120 & $4.0(4.5)$ & $4.7(5.4)$ & - & - & - & - \\
\hline \multirow[t]{6}{*}{ VISTAvis } & $I$ & $1 / 30$ & 60 & $34.5(39.9)$ & $39.2(46.3)$ & $0.3(0.3)$ & - & - & - \\
\hline & & & 90 & $39.8(45.9)$ & $45.6(53.7)$ & $0.3(0.4)$ & - & - & - \\
\hline & & & 150 & $43.2(50.0)$ & 49.7(58.7) & $0.4(0.5)$ & - & - & - \\
\hline & $R$ & $1 / 30$ & 60 & $22.8(26.3)$ & $25.4(29.7)$ & $0.1(0.1)$ & - & - & - \\
\hline & & & 90 & $25.3(29.0)$ & $28.4(33.1)$ & $0.1(0.2)$ & - & - & - \\
\hline & & & 150 & 27.1(31.0) & $30.5(35.5)$ & $0.2(0.2)$ & - & - & - \\
\hline \multirow[t]{3}{*}{ VISTA-IR } & $J$ & $1 / 30$ & 30 & $11.5(13.7)$ & $12.7(15.5)$ & $0.1(0.1)$ & - & - & - \\
\hline & $H$ & $1 / 30$ & 30 & $8.5(10.6)$ & $9.1(11.7)$ & $0.1(0.1)$ & - & - & - \\
\hline & $K$ & $1 / 30$ & 30 & $4.6(6.2)$ & $4.7(6.5)$ & - & - & - & - \\
\hline CFHT & $i^{\prime}$ & $1 / 60$ & 162 & $21.7(24.5)$ & $42.0(48.9)$ & $1.3(1.6)$ & - & - & - \\
\hline SUBARU & $I$ & $1 / 60$ & 120 & $7.5(8.3)$ & $15.2(17.2)$ & $0.6(0.7)$ & - & - & - \\
\hline VISTAvis & $I$ & $1 / 60$ & 150 & $55.1(62.2)$ & $107.4(124.5)$ & $3.4(4.2)$ & - & - & - \\
\hline \multirow[t]{3}{*}{ VISTA-IR } & $J$ & $1 / 60$ & 30 & $15.2(17.6)$ & 28.3(33.9) & $0.7(0.9)$ & - & - & - \\
\hline & $H$ & $1 / 60$ & 30 & $11.9(14.4)$ & $20.9(26.3)$ & $0.5(0.7)$ & - & - & - \\
\hline & $K$ & $1 / 60$ & 30 & $7.0(9.1)$ & $11.3(15.2)$ & $0.3(0.4)$ & - & - & - \\
\hline CFHT & $i^{\prime}$ & $1 / 120$ & 162 & $25.0(27.7)$ & $56.4(64.3)$ & $5.4(6.6)$ & $0.1(0.1)$ & $0.0(0.1)$ & - \\
\hline SUBARU & $I$ & $1 / 120$ & 120 & $8.4(9.1)$ & $19.8(22.0)$ & $2.5(2.9)$ & - & - & - \\
\hline VISTAvis & $I$ & $1 / 120$ & 150 & $63.4(69.9)$ & $143.7(163.3)$ & $13.9(17.0)$ & $0.2(0.2)$ & $0.1(0.2)$ & - \\
\hline \multirow[t]{3}{*}{ VISTA-IR } & $J$ & $1 / 120$ & 30 & $17.9(20.0)$ & $39.1(45.6)$ & $3.0(3.5)$ & - & $0.1(0.2)$ & - \\
\hline & $H$ & $1 / 120$ & 30 & $14.7(17.1)$ & $30.1(36.7)$ & $2.2(2.7)$ & - & $0.1(0.1)$ & - \\
\hline & $K$ & $1 / 120$ & 30 & $9.3(11.7)$ & $17.2(22.6)$ & $1.3(1.7)$ & - & - & - \\
\hline CFHT & $i^{\prime}$ & $1 / 180$ & 162 & $26.3(28.8)$ & $61.0(69.0)$ & $7.8(9.5)$ & $0.1(0.1)$ & $0.0(0.1)$ & - \\
\hline SUBARU & $I$ & $1 / 180$ & 120 & $8.8(9.4)$ & $21.2(23.3)$ & $3.6(4.2)$ & $0.1(0.1)$ & $0.0(0.1)$ & - \\
\hline VISTAvis & $I$ & $1 / 180$ & 150 & $66.6(72.6)$ & $155.3(175.0)$ & $20.1(24.5)$ & $0.3(0.4)$ & $0.1(0.3)$ & $0.0(0.1)$ \\
\hline \multirow[t]{3}{*}{ VISTA-IR } & $J$ & $1 / 180$ & 30 & $18.9(20.9)$ & $42.6(49.1)$ & $4.3(5.1)$ & $0.1(0.1)$ & $0.2(0.3)$ & - \\
\hline & $H$ & $1 / 180$ & 30 & $15.8(18.1)$ & $33.3(40.2)$ & $3.1(3.8)$ & - & $0.1(0.2)$ & - \\
\hline & $K$ & $1 / 180$ & 30 & $10.4(12.7)$ & $19.6(25.4)$ & $1.9(2.4)$ & - & $0.0(0.1)$ & - \\
\hline CFHT & $i^{\prime}$ & $2 / 120$ & 162 & 28.7(30.8) & $70.2(77.8)$ & $16.7(20.2)$ & $0.4(0.5)$ & $0.1(0.2)$ & - \\
\hline SUBARU & $I$ & $2 / 120$ & 120 & $9.4(9.9)$ & $23.7(25.7)$ & $7.4(8.6)$ & $0.2(0.3)$ & $0.1(0.1)$ & - \\
\hline VISTA-Vis & $I$ & $2 / 120$ & 150 & $72.4(77.4)$ & $178.1(196.5)$ & $42.9(51.6)$ & $1.1(1.3)$ & $0.3(0.6)$ & $0.1(0.1)$ \\
\hline \multirow[t]{3}{*}{ VISTA-IR } & $J$ & $2 / 120$ & 30 & $20.9(22.5)$ & $50.1(56.1)$ & $9.4(11.0)$ & $0.2(0.2)$ & $0.3(0.6)$ & $0.0(0.1)$ \\
\hline & $H$ & $2 / 120$ & 30 & $18.0(19.9)$ & $40.9(47.6)$ & $7.1(8.5)$ & $0.1(0.1)$ & $0.2(0.4)$ & - \\
\hline & $K$ & $2 / 120$ & 30 & $12.6(15.0)$ & $25.7(32.3)$ & $4.3(5.4)$ & $0.1(0.1)$ & $0.1(0.1)$ & - \\
\hline CFHT & $i^{\prime}$ & $3 / 120$ & 162 & $30.5(32.1)$ & $77.0(83.8)$ & $24.8(29.5)$ & $1.1(1.3)$ & $0.2(0.4)$ & $0.1(0.1)$ \\
\hline SUBARU & $I$ & $3 / 120$ & 120 & $9.8(10.2)$ & $25.5(27.2)$ & $10.6(12.2)$ & $0.5(0.6)$ & $0.1(0.3)$ & $0.0(0.1)$ \\
\hline VISTAvis & $I$ & $3 / 120$ & 150 & $76.7(80.7)$ & $194.7(211.1)$ & $63.6(75.2)$ & $2.8(3.3)$ & $0.5(1.2)$ & $0.1(0.3)$ \\
\hline \multirow[t]{3}{*}{ VISTA-IR } & $J$ & $3 / 120$ & 30 & $22.2(23.5)$ & $55.5(60.8)$ & $14.0(16.1)$ & $0.5(0.5)$ & $0.6(1.0)$ & $0.1(0.2)$ \\
\hline & $H$ & $3 / 120$ & 30 & $19.6(21.2)$ & $46.7(52.9)$ & $10.7(12.7)$ & $0.3(0.3)$ & $0.4(0.7)$ & $0.1(0.1)$ \\
\hline & $K$ & $3 / 120$ & 30 & $14.6(16.7)$ & $31.2(38.0)$ & $6.6(8.3)$ & $0.2(0.3)$ & $0.1(0.2)$ & - \\
\hline CFHT & $i^{\prime}$ & $4 / 120$ & 162 & $31.5(32.9)$ & $81.1(87.1)$ & $30.7(36.0)$ & $1.8(2.1)$ & $0.3(0.6)$ & $0.1(0.1)$ \\
\hline SUBARU & $I$ & $4 / 120$ & 120 & $10.0(10.3)$ & $26.6(28.0)$ & $12.9(14.6)$ & $0.9(1.0)$ & $0.2(0.4)$ & $0.1(0.1)$ \\
\hline VISTAvis & $I$ & $4 / 120$ & 150 & $79.1(82.4)$ & $204.8(219.3)$ & 78.5(91.7) & $4.6(5.5)$ & $0.8(1.8)$ & $0.2(0.4)$ \\
\hline \multirow[t]{3}{*}{ VISTA-IR } & $J$ & $4 / 120$ & 30 & $23.0(24.0)$ & $58.8(63.4)$ & $17.2(19.7)$ & $0.8(0.9)$ & $0.8(1.5)$ & $0.2(0.2)$ \\
\hline & $H$ & $4 / 120$ & 30 & $20.6(21.9)$ & $50.5(56.1)$ & $13.4(15.7)$ & $0.5(0.6)$ & $0.6(1.0)$ & $0.1(0.2)$ \\
\hline & $K$ & $4 / 120$ & 30 & $15.8(17.8)$ & $35.1(41.8)$ & $8.4(10.4)$ & $0.4(0.4)$ & $0.2(0.3)$ & $0.0(0.1)$ \\
\hline
\end{tabular}



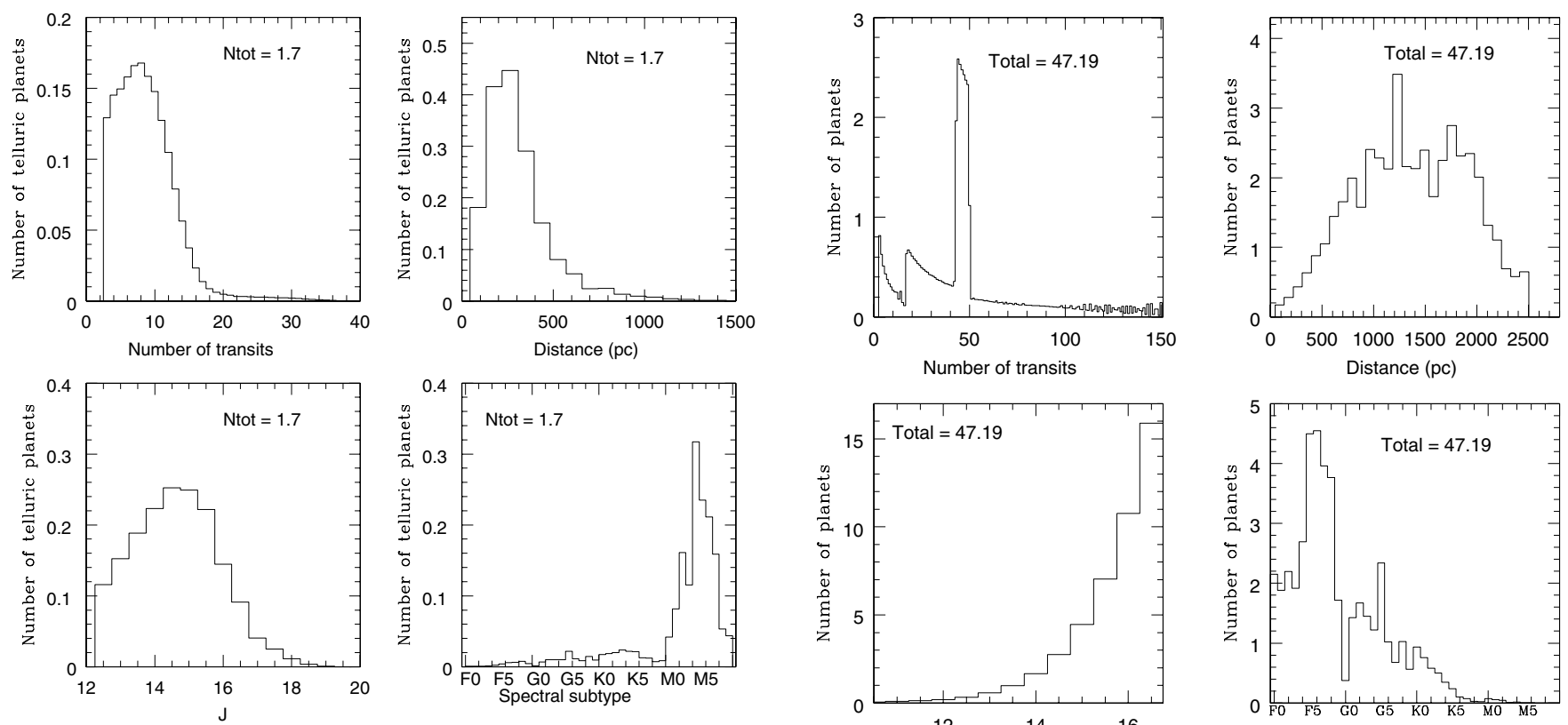

Fig. 7. Distribution of predicted telluric planet discoveries $(\beta=7)$ for VISTA-IR and the 4-years $J$-band survey, as a function of the number of observed transits (upper left), distance (upper right), magnitude (bottom left) and of the host star spectral subtype (bottom right).
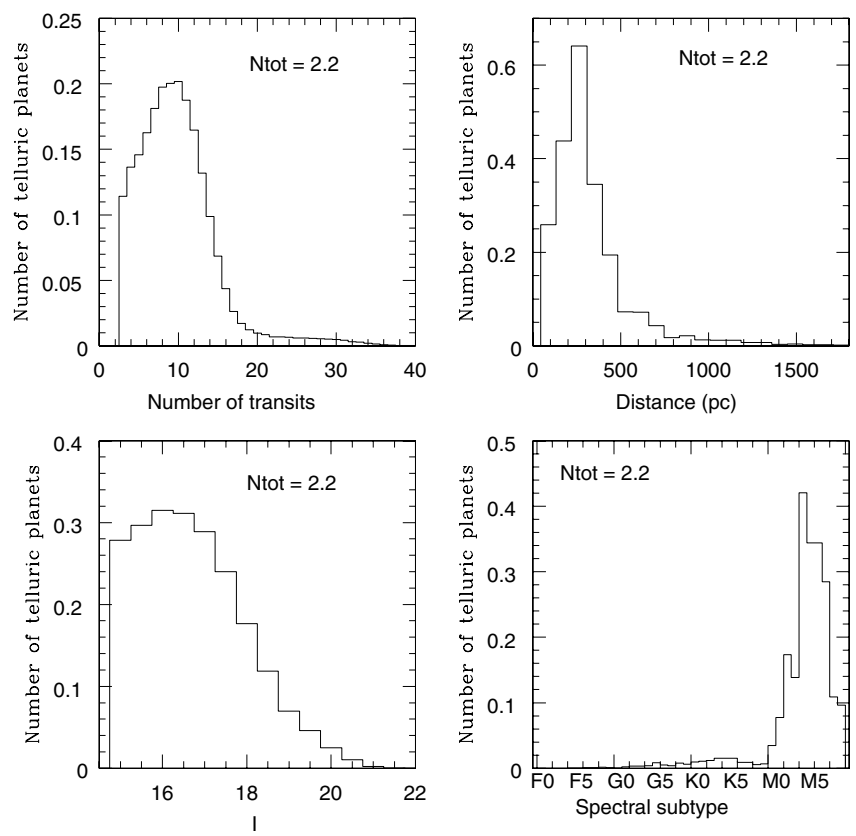

Fig. 8. Same as Fig. 7 but for VISTA-Vis and 4 years of observation through the $I$ filter.
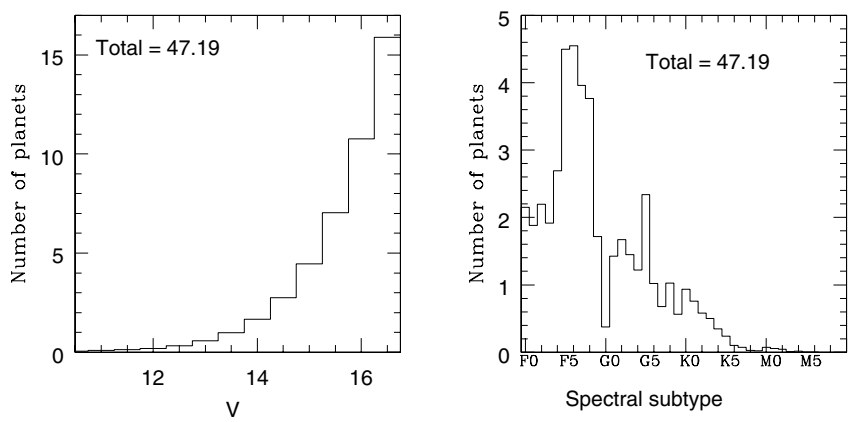

Fig. 9. Distribution of predicted planets (all types included) discoveries using $\beta=7$ for the COROT mission, as a function of the number of observed transits (upper left), distance (upper right), magnitude (bottom left) and of the host spectral subtype (bottom right).
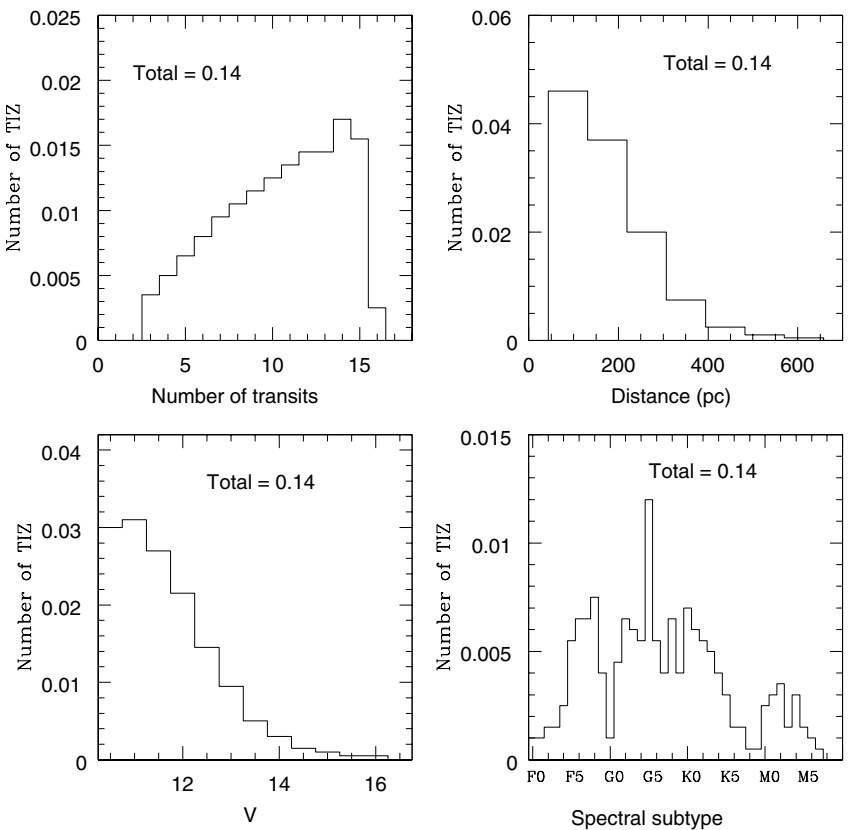

Fig. 10. Same as Fig. 9 but for telluric planets.

between $V=11$ and $V=16.5$. The exposure time will be $31.7 \mathrm{~s}$, without any filter.

We use the expected number of photoelectrons per exposure for every magnitude bin between $V=11$ and $V=16.5$ as well as the different noise contributions given on the COROT web site ${ }^{3}$ and in Bordé (2003) to compute the theoretical SNR per exposure. Using in addition the duration of one field observation (150 days continuously), and multiplying the obtained

\footnotetext{
${ }^{3}$ http://www.astrsp-mrs.fr/projets/exoplan/corot/
}

harvest by 5 to take into account the 5 fields that will be monitored, we compute the harvests presented in Table 5. The distributions of the total number of planets detected for $\beta=7$ as a function of the characteristics of the host stars and of the number of transits observed are shown in Figs. 9 and 10 (TIZ). Figure 11 shows the distribution of the LMSS as a function of their magnitude in the $V$-band, for a typical field observed by COROT. 


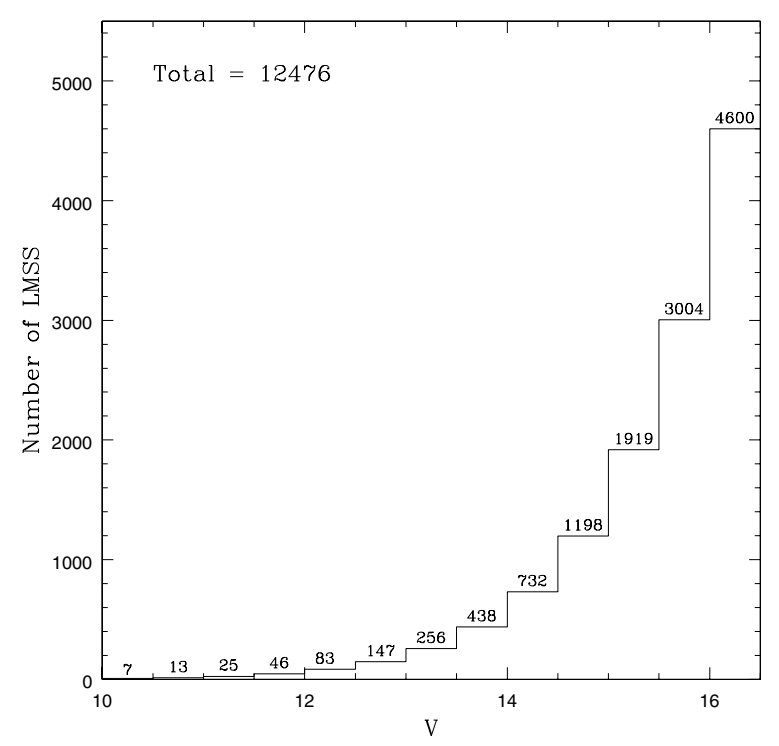

Fig. 11. Magnitude distribution of the $V \leq 16.5$ LMSS in a typical COROT field.

Table 5. Results obtained for COROT, integrated over the duration of the whole mission.

\begin{tabular}{ccccc}
\hline \hline$\beta$ & $V H J$ & $H J$ & $G I Z$ & $T I Z$ \\
\hline 9 & 10.5 & 28.8 & 4.4 & 0.1 \\
7 & 10.7 & 29.5 & 4.6 & 0.1 \\
\hline
\end{tabular}

Bordé et al. (2003) estimated that COROT will be able to detect numerous giant planets, a prediction that is confirmed by our results. It is nonetheless immediately apparent that the expected number of giant planets discoveries $(\sim 45)$ after 2.5 years is well within the reach of ground-based surveys (VISTA-VIS, 30 days) (CFHT, VISTA-IR, 60 days). Only SUBARU would need a survey of several years to be able to compete, due to its smaller field of view.

Bordé et al. (2003) argue that COROT will be able to detect large terrestrial planets ( $R \geq R_{\oplus}$, with $R_{\oplus}=$ earth radius) in close orbits, provided that this kind of object is frequent. Under our assumptions, however, the probability that COROT finds a telluric planet is rather small, even though we only consider telluric planets similar to the ones in our Solar System. The COROT mission will give us informations about the possible existence of "Big Hot Earths". We note, however, that similar harvests might be expected for ground-based surveys as well in reasonable amounts of time, e.g., 4 months with VISTA-VIS or VISTA-IR, or 2 years with the CFHT or with SUBARU.

\subsection{Expected harvest of the KEPLER mission}

The KEPLER mission is dedicated to the detection of earthlike planets orbiting sun-like stars in the $H Z$ (Koch et al. 1998). This satellite is scheduled for launch in 2007, and will observe continuously one single $105 \mathrm{deg}^{2}$ field during 4 years. It will monitor $\sim 100000$ main sequence A-K stars with a mean magnitude of $V=14$ using a $0.95 \mathrm{~m}$ telescope and $3 \mathrm{~s}$ exposures.
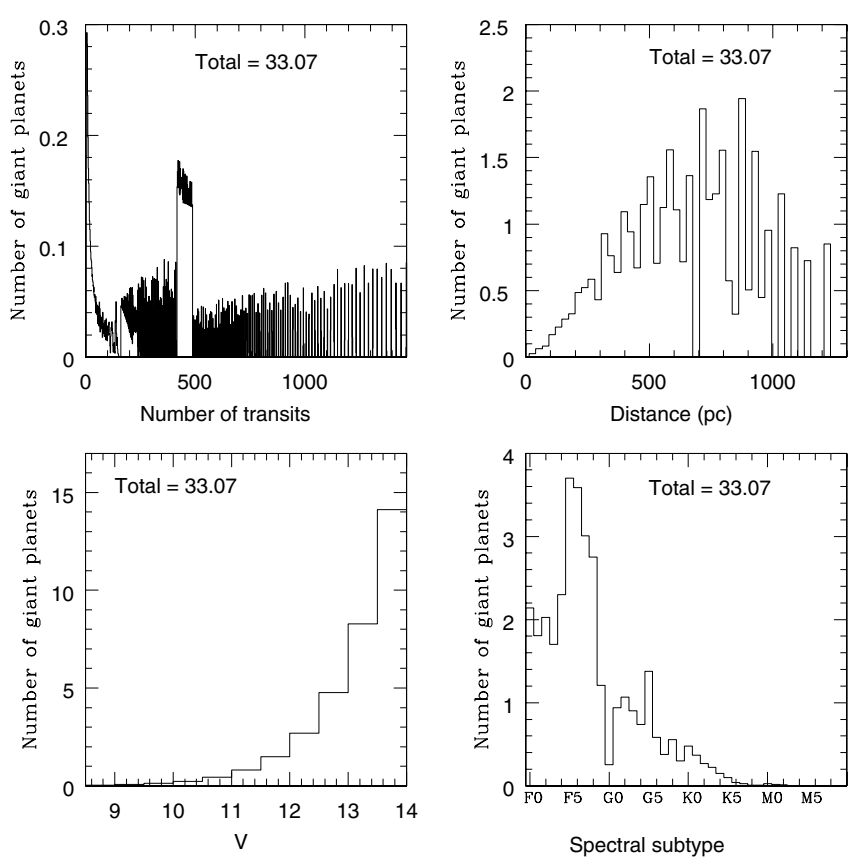

Fig. 12. Distribution of predicted giant planet discoveries using $\beta=7$ for KEPLER, as a function of the number of observed transits (upper left), distance (upper right), magnitude (bottom left) and host star spectral subtype (bottom right).

Table 6. Results obtained for the KEPLER mission.

\begin{tabular}{ccccccc}
\hline \hline$\beta$ & $V H J$ & $H J$ & $G I Z$ & $G H Z$ & $T I Z$ & $T H Z$ \\
\hline 9 & 6.4 & 18.0 & 7.7 & 1.0 & 52.7 & 2.7 \\
7 & 6.4 & 18.0 & 7.7 & 1.0 & 79.4 & 4.6 \\
\hline
\end{tabular}

Using the technical data available on the KEPLER web site ${ }^{4}$, we compute the $S N R$ for an individual exposure time, and use it to estimate the planet harvest of KEPLER under the same assumptions as in the previous sections. KEPLER will observe a field with a mean galactic latitude of $13.3^{\circ}$, leading to a maximal distance $D_{L} \sim 1300 \mathrm{pc}$ according to Eq. (1). The results are presented in Table 6 . Figures 12-14 show the distribution of expected planet discoveries $(\beta=7)$ as a function of the number of observed transits and of the characteristics of their host star.

KEPLER should be able to detect many telluric planets, even in the $H Z$. In contrast, the expected harvest in giant planets is smaller than for COROT, and is also smaller than the one expected for 30-days surveys carried out with VISTA-Vis. This is due to the larger field of view of KEPLER and to its shorter exposure times. This strategy is certainly the best one for hunting telluric planets, but does not allow to observe enough LMSS to detect a large number of giant planets in close orbits.

According to the KEPLER web site, the expected results of the mission are the following:

- assuming that most telluric planets have $R \sim 1 R_{\oplus}, 50$ discoveries should be made. This number increases to 185 if most telluric planets have $R \sim 1.3 R_{\oplus}$ and to 640 if most of them have $R \sim 2.2 R_{\oplus}$.

\footnotetext{
${ }^{4}$ http://www.kepler.arc.nasa.gov
} 

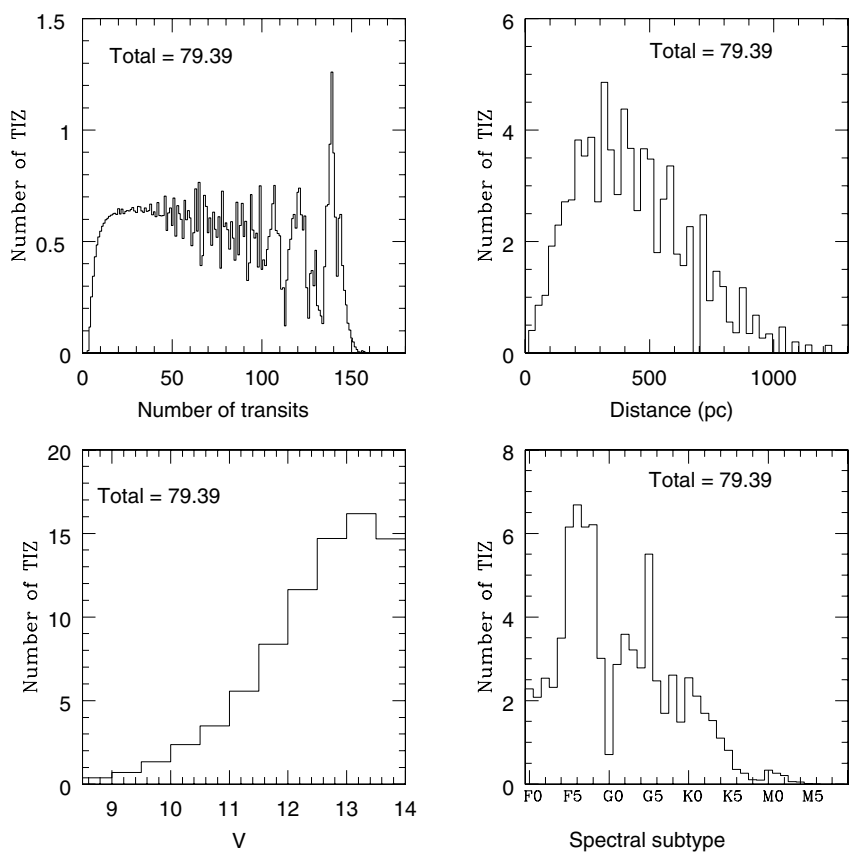

Fig. 13. Same as Fig. 12 but for $T I Z(\beta=7)$.
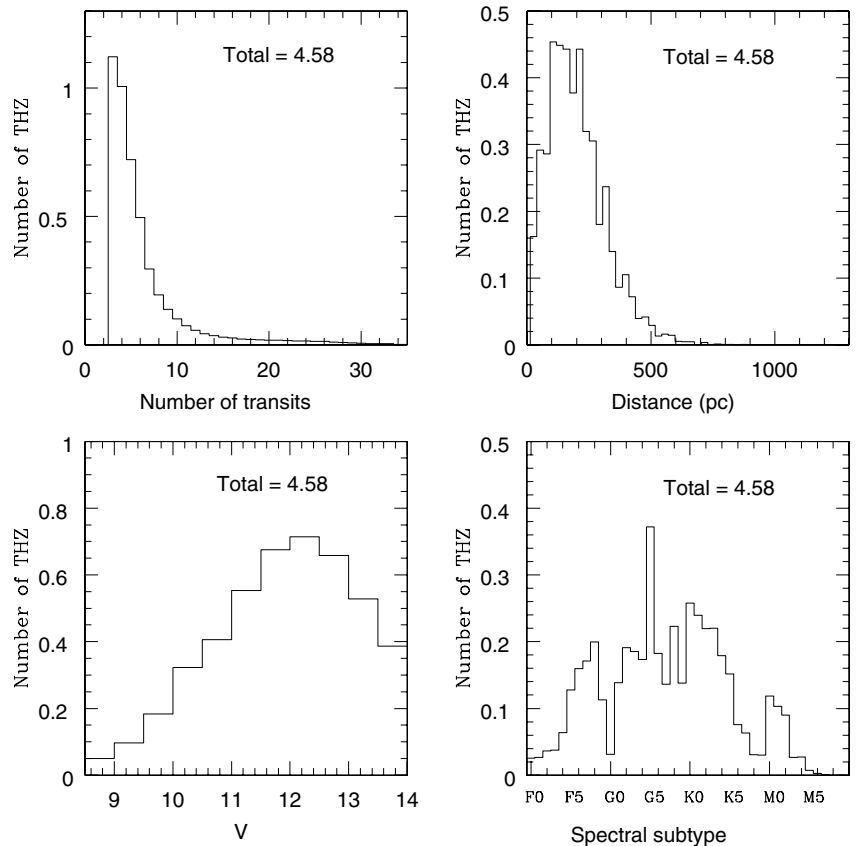

Fig. 14. Same as Fig. 12 but for $T H Z(\beta=7)$.

- 135 inner-orbit giant planets and 30 outer-orbits giant planets should be discovered by KEPLER, plus another 870 $(\mathrm{V}) \mathrm{HJ}$ discovered through the modulation of their reflected light.

These expectations are based on the following assumptions:

- The number of main-sequence stars monitored is $\sim 100000$, after the exclusion of the most active dwarf stars.

- The variability of these $\sim 100000$ stars on the time-scale of a transit is solar or close to solar.

- All stars host in average two earth-size planets in the region between 0.5 and $1.5 \mathrm{AU}$.

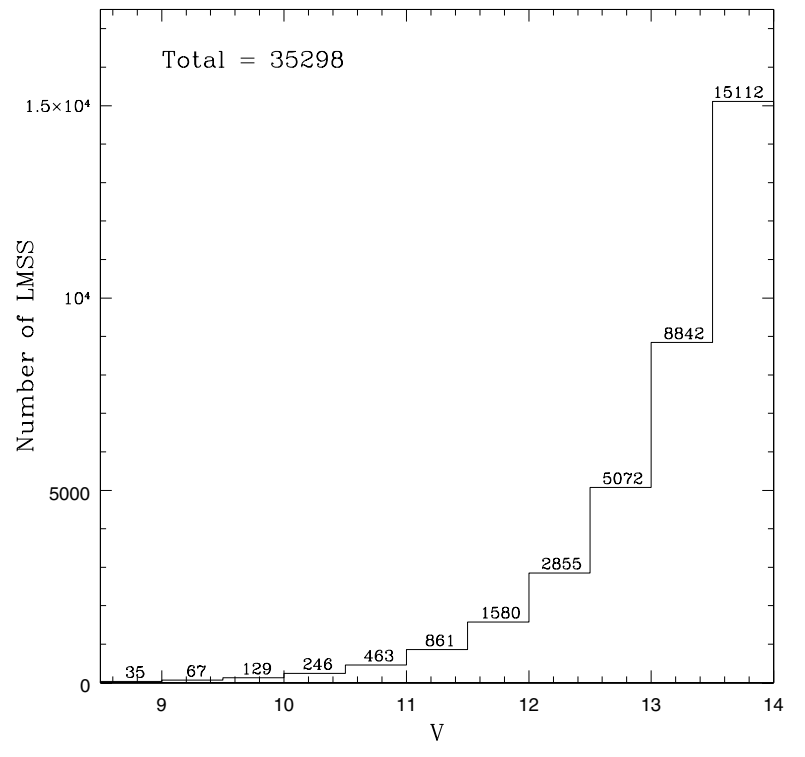

Fig. 15. Magnitude distribution of the $V \leq 14 L M M S$ in the KEPLER field of view.

- Every star host one single giant planet in a jovian-like orbit. - On average, $1 \%$ of the target stars have a giant planet with an orbital period smaller than 1 week, $1 \%$ with periods between 1 week and 1 month and $1 \%$ with a period between 1 month and 1 year.

Detections around binaries are taken into account in these expectations. The detection criterion used by the KEPLER team is $N_{\min }=3, \beta=8$.

Our estimate of the number of LMSS in the KEPLER field leads to a value of $\sim 35000$. Figure 15 shows the distribution of these LMSS as a function of their apparent magnitude. The discrepancy between our number of target stars and the one from the KEPLER team may have two origins: (1) we do not take into account the spectral types $\mathrm{O}, \mathrm{B}$ and $\mathrm{A},(2)$ we assume a constant density of LMSS in a given volume, and nothing outside, (3) the actual extinction coefficient $A_{\mathrm{V}}$ of the KEPLER field should be somewhat lower than $0.7 \mathrm{mag} / \mathrm{kpc}$.

For the giant planets, scaling our result (the sum of the first 4 columns in Table $6, \sim 33$ planets) to the number of targets stars expected by KEPLER and taking into account the possible detection around binaries would lead to a value in good agreement with the one of the KEPLER team.

Our assumptions about telluric planets are quite different from the ones used by the KEPLER team, but our results $(\sim 50-80)$ are in good agreement, provided that the values presented on the KEPLER web site include all telluric planets, and not only habitable ones.

KEPLER is a key project in the search for life outside our Solar System. We can remark from Fig. 14 that habitable planets would be detected mainly around $\mathrm{G}$ and $\mathrm{K}$ dwarfs located about 200 pc from the Sun, thus around nearby solar-type stars. The predicted number of $T H Z(3-5)$ is small. It could become much higher if our radii distribution proved to be too pessimistic. 


\section{Discussion - conclusions}

The main purpose of our simulations was to weight the advantages and disadvantages of various ground- and space-based searches for exoplanets using the transit method. Tables 2 to 6 summarize our results and provide the expected harvest in exoplanets for a broad variety of telescope/instrument combinations. Our main conclusions are the following:

1. As far as telluric planets in the Habitable Zone are concerned, space-based surveys are the only viable option. Such searches remain extremely difficult. They not only require a space instrument, but also a very wide field of view. From space, only the KEPLER mission should be able to find telluric planets in the Habitable Zone.

2. Telluric planets in the Intermediate Zone are much easier to discover. KEPLER could detect more than 50 of them during its four years of observations. Only a few (1-2) TIZ might be discovered from ground-based surveys of the same duration, using VISTA-Vis.

3. Ground-based searches are better than space searches at finding giant planets. While KEPLER is about as efficient as CFHT at finding giant planets in the Habitable Zone (with 1 expected discovery $v s$ 2), a CFHT search is 4 times better than KEPLER at finding the same planets in the Intermediate Zone, and 5 times better for $(V) H J$. This is due to the much deeper exposures.

Ground-based and space-based transit searches are complementary. Because they go deeper, ground-based searches easily find large planets with a short period, such as $(V) H J$. Space searches remain mandatory for telluric planets. Although COROT might find a few telluric planets, we shall have to wait for KEPLER to obtain a significant harvest of such objects, provided that they are not significantly less common than expected.

The above results give orders of magnitude estimates for the expected harvests and allow to emphasize the relative merits and drawbacks of the different searches. However, a word of caution should be given, to avoid over-interpretation of the results. One need to be aware that:

- Space missions like COROT or KEPLER will defocus the images, increasing drastically the size of the Point Spread Function (PSF). While this will minimize saturation of bright objects and increase the $S N R$ per image, it might result in severe image blending. The corresponding loss of efficiency in transit detection will depend on the method used to post-process the data, as do the effects of blends. This is why we have deliberately chosen not to take PSF convolution into consideration and to leave it for a future work. All our estimates are therefore upper limits on the expected harvests.

- The weather simulations used are very simple, and could be somewhat optimistic. Real weather conditions could lead to lower harvests for ground - based surveys.

- The photometric techniques required for the analysis of ground-based surveys are very efficient in the optical (e.g., PSF fitting, image subtraction, image deconvolution), but may be less efficient for near-IR data, where the sky subtraction is more critical. We therefore expect our near-IR harvest estimates to be slightly more optimistic than the optical estimates.

- In the near-IR, second-order extinction effects can have a large impact on the photometric accuracy (Bailer-Jones \& Lamm 2003). Time dependent atmospheric extinction depends on the spectral energy distribution of the target. This may imply the need to use reference objects of the same spectral type as the target stars and complicate further the analysis of near-IR data.

Acknowledgements. The authors would like to thank G. MallénOrnelas for useful discussions and suggestions, and A. Udalski for providing informations about OGLE-III. We acknowledge financial support from the Prodex-ESA Contract 15448/01/NL/Sfe(IC).

\section{References}

Alonso, R., Brown, T. M., Torres, G. et al. 2004, ApJ, 613, L153

Baglin, A., et al. 1998, Asteroseismology from space - The COROT experiment, in New Eyes to See Inside the Sun and Stars, IAU, 185,301

Bailer-Jones, C. A. L., \& Lamm, M. 2003, MNRAS, 339, 477

Binney, J., \& Merrifield, M. 1998, galactic Astronomy (Princeton University Press)

Bordé, P. 2003, Ph.D. Thesis, École doctorale Astronomie \& Astrophysique d'Ile-de-France, Observatoire de Paris, Université Pierre et Marie Curie

Bordé, P., Rouan, D., \& Léger, A. 2003, A\&A, 405, 1137

Bouchy, F., Pont, F., Santos, N. C., et al. 2004, A\&A, 421, L13

Bouchy, F., Pont, F., Melo, C., et al. 2005, A\&A, 431, 1105

Butler, R. P., et al. 2000, in Planetary Systems in the Universe: Observation, Formation, and Evolution, ed. A. Penny, P. Artymowicz, A.-M. Lagrange, \& S. Russell (San Fransisco: ASP), IAU Symp., 202, in press

Chambers, J. E. 2001, Icarus, 152, 205

Charbonneau, D., Brown, T. M., Latham, D. W., \& Mayor, M. 2000, ApJ, 529, L45

Gaudi, B. S., Seager, S., \& Mallén-Ornelas, G. 2005, ApJ, 623, 472

Gliese, W., \& Jarheiss, H. 1991, Preliminary Version of the Third Catalogue of Nearby Stars, Astron. Rechen-Institut, Heidelberg

Henry, G. W., Marcy, G. W., Butler, R. P., \& Vogt, S. S. 2000, ApJ, 529, L41

Kasting, J. F., Whitmire, D. P., \& Reynolds, R. T. 1993, Icarus, 101, 108

Koch, D., et al. 1998, SPIE Conference 3356, Space Telescope and Instruments V, 599

Konacki, M., Torres, G., Jha, S., \& Sasselov, D. D. 2003a, Nature, 421, 507

Konacki, M., Torres, G., Sasselov, D. D., \& Jha, S. 2003b, ApJ, 597, 1076

Konacki, M., Torres, G., Sasselov, D. D., \& Jha, S. 2005, ApJ, in press

Mallén-Ornelas, G., Seager, S., Yee, H. K. C., et al. 2003, ApJ, 582, 1123

Mandushev, G., Torres, G., Latham, D. W., et al. 2005, ApJ, 621, 1061

Marcy, G. W., Butler, R. P., Fischer, D. A., \& Vogt S. S. 2003, ASP Conf. Ser., 294

Mayor, M., \& Queloz, D. 1995, Nature, 378, 355

Perryman, M. A. C. 2000, Rep. Prog. Phys., 63, 1209

Pont, F., Bouchy, F., Queloz, D., et al. 2004, A\&A, 426, 15 
Rouan, et al. 2000, Detecting Earth-Uranus class planets with the space mission COROT. In Darwin and Astronomy - The Infrared Space Interferometer, ESA SP-451, 221

Santos, N. C., Israelian, G., \& Mayor, M. 2004, A\&A, 415, 1153

Sackett, P. D., 1999, in Planets outside the Solar System: Theory and Observations (NATO-ASI), ed. J. M. Mariotti \& D'Alloin (Dordrecht: Kluwer), 189

Sato, B., et al. 2005, ApJ, accepted

Schlegel, D. J., Finkbeiner, D. P., \& Davis, M. 1998, ApJ, 500, 525

Schneider, J. 2000, in VLT Opening Ceremony Symposium, ed. F. Paresce (Berlin: Springer)
Schneider, J. 1996, Extrasolar Planet Encyclopaedia

Udalski, A., Paczynski, B., Zebrun, K., et al. 2002a, Acta Astron., 52, 1

Udalski, A., Zebrun, K., Szymanski, M., et al. 2002b, Acta Astron., 52,115

Udalski, A., Szymanski, M. K., Kubiak, M., et al. 2004, Acta Astron., 54,313

Udry, S., Mayor, M. Clausen, J. V., et al. 2003, A\&A, 407, 679

Zakhozhaj, V. A., 1979, Catalogue of nearest stars until 10 pc, Vestnik Khar'kovskogo Universiteta 190, 52 